\title{
PRODUÇÃO DE MUDAS DE ACEROLA (Malpighia emarginata D.C) PELO MÉTODO DE ENXERTIA EM TOPO POR GARFAGEM EM FENDA CHEIA
}

\author{
Aelton dos Santos Bezerra ${ }^{1}$; James Luan Noleto Leite ${ }^{2}$; Kalyne Rosa da Silva ${ }^{3}$; Igor Vinicius \\ de Oliveira4; Andréa Hentz de Mello ${ }^{5}$. \\ ${ }^{1}$ Universidade Federal do Sul e Sudeste do Pará (UNIFESSPA), Marabá, Pará, Brasil, \\ aeltonsantosmaraba@hotmail.com \\ 2 UNIFESSPA, Marabá, Pará, Brasil, luan.jld@gmail.com \\ ${ }^{3}$ UNIFESSPA, Marabá, Pará, Brasil, kalynerosa_agro@hotmail.com \\ ${ }^{4}$ UNIFESSPA, Marabá, Pará, Brasil, igor.oliveira@unifesspa.edu.br \\ ${ }^{5}$ UNIFESSPA, Marabá, Pará, Brasil, andreahentz@unifesspa.edu.br
}

RESUMO: A fruticultura possui elevado efeito multiplicador de renda e, portanto, com força suficiente para dinamizar economias. A acerola vem ganhando espaço na fruticultura, com a crescente demanda faz necessária à obtenção de plantas que inicie a produção em menor tempo, podendo ser alcançado com produção de mudas vigorosas. A enxertia é o processo que une duas plantas, uma contribuindo com o sistema radicular, denominada portaenxerto, e outra contribui com a parte aérea de fundamental importância para obtenção de mudas vigorosas. O objetivo deste trabalho foi avaliar a eficiência do método de propagação enxertia por garfagem no topo em fenda cheia, em mudas de acerola em condições de casa de vegetação. O experimento foi conduzido em casa de vegetação na Faculdade de Ciências Agrárias de Marabá, unidade II, da Universidade Federal do Sul e sudeste do Pará, sendo 205 mudas de acerola, sem distinção de espécies, submetidas ao processo de enxertia por garfagem em fenda cheia. A enxertia por garfagem no topo em fenda cheia é indicada para ser utilizada na propagação da acerola. O tempo para as mudas estarem aptas a irem a campo foi de 12 meses, com boas condições como altura e diâmetro.

PALAVRAS-CHAVE: Fruticultura, Propagação, Tecnologia.

\section{PRODUCTION OF ACEROLA SEEDLINGS (Malpighia emarginata D.C) BY THE GRAFIING METHOD FOR FULL SLOTTED TOP}

ABSTRACT: Fruit cultivation has a high income multiplier effect and, therefore, with enough
force to boost economies. The acerola has been gaining space in the fruit growing, with the
increasing demand makes necessary to obtain plants that start the production in a shorter
time, being able to be reached with vigorous seedlings production. Grafting is the process
that joins two plants, one contributing to the root system, called rootstock, and another
contributing to the aerial part of fundamental importance for obtaining vigorous seedlings.
The objective of this work was to evaluate the efficiency of the grafting propagation method
in the slotted top, in acerola seedlings under greenhouse conditions. The experiment was
conducted in a greenhouse at the Faculty of Agrarian Sciences of Marabá, unit II, Federal
University of Southern and Southeastern of Pará, being 205 acerola seedlings, without 
distinction of species, submitted to the process of grafting by grafting in a full slot. Grafting by grafting at the top in a full slot is indicated for use in the propagation of acerola. The time for the seedlings to be able to go to the field was 12 months, with good conditions such as height and diameter.

KEYWORDS: Fruticulture, Propagation, Technology.

\section{PRODUCCIÓN DE MUDAS DE ACEROLA (Malpighia emarginata D.C) POR EL MÉTODO DE INJERTO EN LA PARTE SUPERIOR POR TAJADO EN RANURA COMPLETA}

RESUMEN: La fruticultura tiene un elevado efecto multiplicador de renta y, por lo tanto, con fuerza suficiente para dinamizar economías. La acerola viene ganando espacio en la fruticultura, con la creciente demanda hace necesaria la obtención de plantas que inicie la producción en menor tiempo, pudiendo ser alcanzado con producción de mudas vigorosas. La injerto es el proceso que une dos plantas, una contribuyendo con el sistema radicular, denominado porta-injerto, y otra contribuye con la parte aérea de fundamental importancia para la obtención de mudas vigorosas. El objetivo de este trabajo fue evaluar la eficiencia del método de propagación injerto por tajado en la cima en hendidura llena, en mudas de acerola en condiciones de casa de vegetación. El experimento fue conducido en casa de vegetación en la Facultad de Ciencias Agrarias de Marabá, unidad II, de la Universidad Federal del Sur y del sudeste de Pará, siendo 205 mudas de acerola, sin distinción de especies, sometidas al proceso de. en el caso de que se trate de un frasco en ranura completa. La injerta por tostado en la parte superior en ranura completa está indicada para ser utilizada en la propagación de la acerola. El tiempo para que las mudas estén aptas para ir al campo fue de 12 meses, con buenas condiciones como altura y diámetro.

PALABRAS CLAVE: Fruticultura, Propagación, Tecnología.

A atividade frutícola possui elevado efeito multiplicador de renda e, portanto, com força suficiente para dinamizar economias locais estagnadas e com poucas alternativas de desenvolvimento (BUAINAIN; BATALHA, 2007). Sendo um dos principais setores que geram perspectivas de desenvolvimento do meio rural, principalmente para agricultura familiar.

Os frutos são conhecidos pelo seu valor nutricional, que trazem benefícios para as pessoas, que as consomem tanto na forma in natura como processada. 
Nesse contexto, a acerola (Malpighia emarginata D.C) vem ganhando espaço na fruticultura, apresentando elevada quantidade de vitamina $C$, inclusive superior a laranja. A aceroleira apresenta grande potencial de expansão, pois o seu uso atende a diversos mercados, seja para o consumo in natura, indústria de sucos ou para a indústria farmacêutica (ALMEIDA et al., 2014).

No Brasil, seu cultivo foi intensificado no período de 1988 a 1992, em virtude da sua importância para a alimentação humana, em função da riqueza em vitamina C, estimada entre 1200 a 1900mg em $100 \mathrm{~g}$ de polpa (PAIVA et al., 2003). A fruticultura participa diretamente na economia do País, através do valor das exportações e mercado interno, e a importância no caráter econômico-social, uma vez que está presente em todos os estados brasileiros (FACHINELLO et al., 2011).

Segundo Godoy et al. (2008) o abastecimento de acerola no mercado pode ser considerado como difícil pela fragilidade dos frutos, que são bastante perecíveis, mas isso não interfere de forma acentuada na crescente demanda.
De acordo com Dantas et al. (2014) a base da fruticultura moderna é a produção de mudas de alta qualidade, e mudas bem formadas dando origem a plantas com alto potencial produtivo.

Sendo a enxertia uma importante técnica para obtenção dessas mudas, para Lima e Cunha (2004) a enxertia é o processo que une duas plantas, uma contribuindo com o sistema radicular, denominada porta-enxerto, e outra contribui com a parte aérea e consequentemente com a frutificação, denominada enxerto.

Segundo Jacomino (2008) as razoes para o uso da enxertia esta nos benefícios como, mudança de cultivar em plantas adultas, substituição do porta-enxerto, vigor da planta, precocidade da produção. Além disso, a presença da raiz pivotante da muda enxertada, confere uma maior sustentação da planta no solo, explorando maior volume do solo (GONZAGA NETO, 1995).

Neste sentido, o objetivo deste trabalho foi avaliar a eficiência do método de propagação enxertia por garfagem no topo em fenda cheia, em mudas de 
acerola em condições de casa de vegetação.

O experimento foi conduzido em casa de vegetação na Faculdade de Ciências Agrárias de Marabá (FCAM), na unidade II, do campus de Marabá-PA, da Universidade Federal do Sul e Sudeste do Pará (UNIFESSPA).

As sementes para produção das mudas de acerola foram adquiridas de plantio de agricultores, que fornecem para Secretaria de Agricultura do Município de Marabá - PA (SEAGRI). Para a produção das mudas de acerola, o fruto foi beneficiado para retirada da semente, posteriormente lavada e deixada secar, antes de ser semeada na sementeira contendo areia lavada.

Aos 30 dias foram repicadas para sacos de polipropileno de $22 \times 15 \mathrm{~cm}$, contendo serragem, esterco de gado curtido, terra preta e carvão, na proporção de 2:1:1:1 e na mistura foram acrescentados calcário e fósforo em proporções não reveladas pela SEAGRI.

Com três meses de idade as mudas foram doadas pela SEAGRI, para a FCAM sendo 205 mudas de acerola, sem distinção de variedade, levadas para casa de vegetação, para serem avaliados os parâmetros de crescimento como altura, diâmetro e porcentagem de pegamento quando feito a enxertia por garfagem no topo em fenda cheia.

As avaliações como altura e diâmetro, foram realizadas com objetivo de verificar a qualidade e uniformidade das mudas, bem como identificar o melhor período para realização da enxertia. A primeira avaliação feita aos cinco meses, utilizando trena e paquímetro respectivamente para as medições. A segunda avaliação ocorreu aos oito aos oito meses, sendo os parâmetros avaliados foram os mesmos, considerando agora os parâmetros favoráveis para a realização do processo de enxertia por garfagem em fenda cheia.

Quando as plantas estavam com 6 meses, foram realizadas quatro aplicações de adubo foliar, sendo que as três primeiras com intervalo de sete dias, a ultima aplicação com intervalo de 15 dias. Nas duas primeiras aplicações foram usados $6 \mathrm{~mL}$ por litro e nas duas últimas 3 $\mathrm{mL}$ por litro de água.

A composição química do produto aplicado, de acordo com fabricante, é de: nitrogênio (N) 7\%; fósforo $\left(\mathrm{P}_{2} \mathrm{O}_{5}\right)$ 3\%; 
potássio $\left(\mathrm{K}_{2} \mathrm{O}\right)$ 9\%; magnésio (Mg) 0,5\%; cálcio (Ca) 1\%; boro (B) 0,2\%; cobre (Cu) 0,08\%; ferro (Fe) 0,1\%; manganês ( $\mathrm{Mn}$ ) 0,2\%; molibdênio (Mo) 0,05\%; Zinco (Zn) $0,4 \%$.

Foi também realizado uma adubação via solo, aos sete meses, com 0,07g de ureia por planta e 0,13g de cloreto de potássio por planta, com intervalo de três dias, o cloreto de potássio parcelado em duas vezes com intervalo de quatro dias, similar ao utilizado por (BOLOTA et al., 2011). Para facilitar a aplicação foram diluídos em um e dois litros de água respectivamente e com uma seringa feita aplicação de 4,89 mL por planta.

Todas as plantas foram conduzidas em haste única, retirando as brotações de forma manual até atingirem porte ideal para serem submetidas à enxertia. O controle de cochonilha e pulgão foi realizado com $50 \mathrm{~g}$ de sabão de coco e 10 g de fumo, em $5 \mathrm{~L}$ de água, o sabão foi diluído em 4L de água, o fumo em $1 \mathrm{~L}$ de água em repouso por 24h, sendo a aplicação realizada com bomba costal de 5L, sempre que necessário, junto com o controle mecânico que consistiu em passar o dedo levemente na folha atacada.

O enxerto utilizado foi decorrente de partes de plantas já produtivas da BRS Jaburu, adquiridas no município de Nova Ipixuna no Estado do Pará, a $60 \mathrm{~km}$ da Cidade de Marabá. Os galhos foram coletados ao fim da tarde, armazenados em caixa de isopor contendo jornal umedecido com água e isolada com fita adesiva, após 12 horas iniciou o processo de enxertia nas mudas, ilustrado na Figura 1.

Os porta-enxertos ao atingiram aproximadamente $30 \mathrm{~cm}$ altura e diâmetro superior a 3 mm, aos oito meses, foram decapitados a $13 \mathrm{~cm}$ de altura e aberto uma fenda para ser inserido o enxerto com a variedade BRS Jaburu, com $15 \mathrm{~cm}$ de altura de três a seis gemas com diâmetro aproximado do porta enxerto.

De acordo com Mora et al. (2010) a junção deve ser realizada com fita plástica, evitando deixar espaços vazios e qualquer entrada de água nos cortes, como mostra a Figura 2. 
Figura 1. Coleta de galhos ponteiro de plantas produtivas e com características desejáveis, armazenamento e conservação.

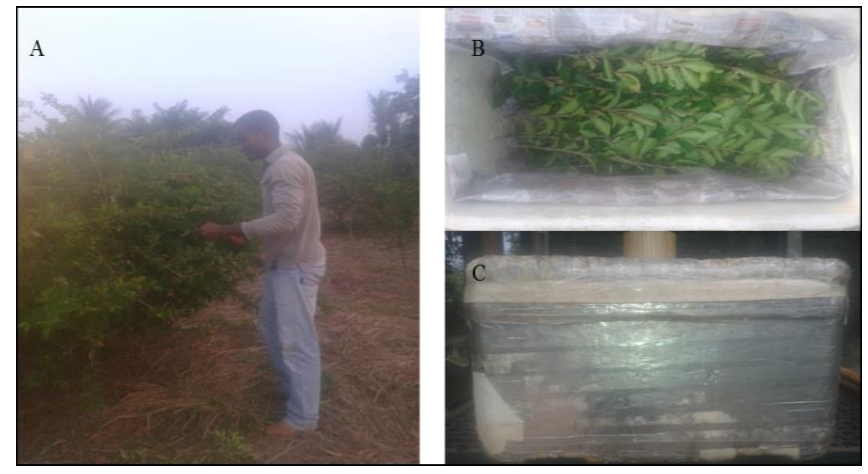

Fonte: Santos (2016).

Figura 2. Processo de enxertia por garfagem em fenda cheia da acerola, amarração e proteção com saco plástico, Unifesspa, Marabá - PA.



Fonte: Santos (2016).

Aos vinte dias após a enxertia foi retirada a câmara úmida, sendo que para garantir a sobrevivência, algumas foram retiradas após os vinte dias. As plantas foram conduzidas em haste única, até atingir a altura adequada para posteriormente serem transplantadas em campo.
Os parâmetros avaliados foram sistematizados em planilhas de excel e observados através da estatística descritiva.

A porcentagem das mudas que pegaram após a enxertia foi de 89,27\%, sendo superior a encontrada por Araújo e Minami (1994), no qual observaram índice de pegamento de até 82,5\% em mudas 
enxertadas de acerola por garfagem no topo em fenda cheia com seis meses de idade, sem informação da variedade do porta-enxerto. O método de enxertia por garfagem no topo em fenda cheia apresentou os seguintes percentuais de pegamento como mostra a Tabela 1.

Tabela 1. Valores médios de pegamento da enxertia (PE), número de enxertos realizados (NER) e número de enxertos vivos (NEV) aos 30 dias após o processo de enxertia.

\begin{tabular}{llll}
\hline Tratamento & NER & NEV & PE (\%) \\
\hline T1 Garfagem em fenda cheia & 205 & 184 & 89,27 \\
\hline
\end{tabular}

Das 205 mudas em que foram submetidas a enxertia, 184 pegaram, mostrando que mesmo sendo um número alto de plantas a taxa de pegamento foi satisfatório. Corroborando com Cardoso et al. (2010) que avaliando diferentes métodos de enxertia na produção de mudas de acerola (Malphigia emarginata D.C) concluiu que a enxertia de garfagem no topo em fenda cheia é a mais indicada para ser utilizada na propagação desta espécie.

Pedrosa et al. (1994) verificaram pegamentos de 65,5\%, quando utilizaram porta-enxertos formados por sementes de variedades não identificadas de acerola. A baixa taxa de pegamento encontrada por esses autores não corroboram com o resultado encontrado neste estudo.
Nasser (2013) avaliando a propagação por garfagem da acerola cv. Okinawa sobre diferentes variedades de porta-enxertos concluiu que não houve diferença entre as combinações enxerto/porta-enxertos quanto ao desenvolvimento inicial das mudas.

O tempo levado para realização do processo da enxertia pode estar diretamente relacionado com a taxa de enxertos pegos. Visto que, as primeiras 50 mudas tiveram 100\% de sobrevivência, já as 50 ultima somente $70 \%$ sobrevivência, sendo que corresponde mais da metade das mudas que não pegaram em todo o processo, desse modo, para o sucesso no processo da enxertia deve-se buscar o menor tempo possível, na Figura 3 podem ser observadas as mudas após a enxertia. 
Figura 3. Mudas de acerola, cultivar BRS Jaburu, aos 60 dias após a enxertia, Marabá - PA.



Fonte: Santos (2016).

Para avaliar o desenvolvimento do enxerto aos 120 dias após o pegamento das mudas, estas foram retiradas da bancada do viveiro e transferidas para o piso, como mostra figura (B), sendo dispostas de modo que todas pudessem estar expostas a radiação solar mais uniforme possível, para ser realizada a avaliação de altura e diâmetro como mostra a Tabela 2.

Tabela 2. Valores médios da altura e diâmetro do caule da acerola aos 120 dias após a enxertia, Marabá - PA.

\begin{tabular}{lcc}
\hline Enxerto BRS Jaburu & Altura $(\mathrm{cm})$ & Diâmetro do coleto $(\mathrm{mm})$ \\
\hline Média & 37,28 & 3,71 \\
Desvio padrão & 15,71 & 0,97 \\
\hline
\end{tabular}

Foi observado que a altura das plantas de $37,28 \mathrm{~cm}$ apresentando um crescimento vigoroso e que a enxertia apresentava ter sido satisfatória, sem prejuízo quanto ao crescimento inicial, por autoincompatibilidade ou qualquer outro fator.
A enxertia por garfagem no topo em fenda cheia mostrou ser eficiente no processo de produção de mudas. Por meio deste método foi possível alcançar o tempo de 12 meses para o transplante de mudas com boas condições de desenvolvimento e altura e diâmetro satisfatórios. 


\section{AGRADECIMENTOS}

Os autores agradecem a PROPIT PIBIC - UNIFESSPA pela bolsa concedida.

\section{REFERÊNCIAS}

ALMEIDA, J. P. N.; DANTAS, L. L. G. R. Fungo micorrízico arbuscular e extrato de algas no crescimento inicial de portaenxerto de aceroleira. Revista de Ciências Agrárias, Fortaleza, v. 57, n. 1, p. 22-28, 2014.

ALVES, R. E.; BEZERRA, M. A.; MIRANDA, F. R.; SILVA, H. Acerola. In: CRISÓSTOMO, L. A.; NAUMOV, A. (Orgs.). Adubando para alta produtividade e qualidade: fruteiras tropicais do Brasil. Fortaleza: Embrapa Agroindústria Tropical, 2009, p. $13-30$

ARAÚJO, P. S. R.; MINAMI, K. Acerola. Campinas: Fundação Cargill, 1994. 81 p.

BOLOTA, E. L.; MACHINESKI, O.; STENZEL, N. M. C. Solos e Nutrição de Plantas: Resposta da acerola à inoculação de fungos micorrízicos arbusculares em solo com diferentes níveis de fósforo. Bragantia, Campinas, v. 70, n. 1, p. 166-175, 2011.

BUAINAIN, A. M.; BATALHA, M. O. Cadeia produtiva de frutas. Brasília: IICA/MAPA/SPA, 2007. v.7, 102 p.

CARDOSO, E. de A.; SILVA, R. M.; AGUIAR, A. V. M.; ARAGÃO, R. G. Métodos de enxertia na produção de mudas de acerola (Malphigia emarginata D.C). Agropecuária Científica no Semi-Árido, Mossoró, v. 06, n 04, p. 28 - 32, 2010.
DANTAS, K. A.; FIGUEIREDO, T. da C:; MESQUITA, E. F.; SILVA SÁ, F. V.; FERREIRA, N. M. Substratos e doses de biofertilizante bovino na produção de mudas de aceroleira. Revista Verde, Mossoró, v. 9 , n. 1, p. 157 - 162, 2014.

FACHINELLO, J. C.; PASA, M. da S.; SCHMTIZ, J. D.; BETEMPS, D. L. Situação e perspectivas da fruticultura de clima temperado no brasil. Rev. Bras. Frutic, Jaboticabal, Volume Especial, p. 109-120, 2011.

GONZAGA NETO, L. Propagação vegetativa: enxertia em aceroleira. In: SÃO JOSÉ, A.B.; ALVES. R.L. Acerola no Brasil: produção e mercado. Vitória da Conquista, BA, DFZ/UESB. 1995. p. 2-46.

JACOMINO. A. P. Enxertia de plantas frutíferas. Disponível em: < https://edisciplinas.usp.br/pluginfile.php/ 3190466/mod_resource/content/1/Aula\% 20Enxertia\%202017-web.pdf >. Acesso em: 13 jul. 2017.

LIMA, A. de A.; CUNHA, M. A. P. Maracujá: Produção e qualidade na passicultura. Cruz das Almas: EMBRAPA MANDIOCA E FRUTICULTURA, 2004. 396p.

MORA, A. L.; BERTOLOTI, G.; HIGA, A. R. Guia prático: Propagação vegetativa de pinus por enxertia. Série (Circular Técnico, n. 42). Disponível em: $<$ www.ipef.br/publicacoes/ctecnica/nr042 .pdf >. Acesso em: 13 jul. 2017.

NASSER, M. D. Propagação por garfagem da aceroleira cv. Okinawa sobre diferentes porta-enxertos. 2013. 51f. Dissertação (mestrado em sistemas de produção) Universidade Estadual Paulista, Faculdade de Engenharia de Ilha Solteira, 2013. 
PAIVA, J. R.; ALVES, R. E.; BARROS, L. M.; CRISÓSTOMO, J. R.; MOURA, C. F. H.; ALMEIDA, A. S. Seleção de clones de acerola (Malpighia emarginata) no Estado do Ceará, Brasil. Proc. Interamer. Soe. Trop. Hort., v. 47, p. 99-102, 2003.

PEDROSA, A.C.; FREITAS, E.V.; LEDERMAN, I.E.; BEZERRA, J.E.F. Influência do processo de enxertia por garfagem na propagação da aceroleira em Pernambuco. In: CONGRESSO BRASILEIRO DE FRUTICULTURA, 13. Salvador. Anais.. Salvador: Sociedade Brasileira de Fruticultura, 1994. v. 1, p. 7879. 